\title{
Health Monitoring for a Structure Using Its Nonstationary Vibration
}

\author{
Yoshimutsu Hirata, ${ }^{1}$ Mikio Tohyama, ${ }^{1}$ Mitsuo Matsumoto, ${ }^{1}$ and Satoru Gotoh ${ }^{2}$ \\ ${ }^{1}$ SV Research Associates, 23-4-304, Yuigahama 2-chome, Kamakura-shi, Kanagawa 248-0014, Japan \\ ${ }^{2}$ Waseda University, 1-104 Totsukama, Shinjuku-ku, Tokyo 169-8050, Japan \\ Correspondence should be addressed to Mitsuo Matsumoto, matsu_desk@jcom.home.ne.jp
}

Received 26 May 2010; Accepted 4 August 2010

Academic Editor: K. M. Liew

Copyright (C) 2010 Yoshimutsu Hirata et al. This is an open access article distributed under the Creative Commons Attribution License, which permits unrestricted use, distribution, and reproduction in any medium, provided the original work is properly cited.

\begin{abstract}
The frequency distribution of a short interval period, the SIP distribution, obtained from the vibration of a structure that is excited by the force of non-stationary vibration is available for the robust estimation of the dynamic property of the structure. This paper shows experiments of the health monitoring of a model structure using the SIP distribution. Comparisons of SIP distributions with average DFT spectra are also shown.
\end{abstract}

\section{Introduction}

One of the major problems after an earthquake, which causes certain changes in the dynamic property of a structure, is the investigation of structural damage. In principle, we can check the change using a shaker to obtain the frequency response of a structure. However, it is difficult and not practical to shake a big structure before and after an earthquake to detect the change. Regardless of size or weight, all structures such as buildings, towers, and bridges are vibrating due to the natural force of winds, ground motions, or both. Information of the dynamic property of a building, for example, is comprised of the forced vibration of a structure. Changes of dynamic property reflect structural changes. Then, the problem is how to extract information from the vibration of structures.

It was shown by one of the authors that the frequency distribution of a short-interval period, the SIP distribution, of the forced nonstationary vibration corresponds to the frequency response of a structure [1]. In this paper, experiments of health monitoring of a model structure using its nonstationary vibrations are shown, where SIP distributions are used for detecting the changes of the dynamic property of a structure.

\section{SIP Distributions}

The discrete Fourier transform (DFT) is used in many disciplines to obtain the spectrum or frequency of a signal. The DFT produces reasonable results for a large class of signal processes. However, we do not use the DFT for detecting a short interval period because of its inherent limitation of frequency resolution [2].

The dominant frequency or period of a short-interval sequence $W(m)(m=0,1, \ldots, M)$ can be given by the nonharmonic Fourier analysis [3]. In the process of the analysis, we put $W(x)=W(m)$, where $x=m-M / 2$, and obtain Fourier coefficients $a(f)$ and $b(f)$ for an arbitrary frequency such that

$$
\begin{aligned}
& a(f)=\frac{\sum_{x=-M / 2}^{M / 2} W(x) \sin (2 \pi f x)}{\sum_{x=-M / 2}^{M / 2} \sin ^{2}(2 \pi f x)}, \\
& b(f)=\frac{\sum_{x=-M / 2}^{M / 2} W(x) \cos (2 \pi f x)}{\sum_{x=-M / 2}^{M / 2} \cos ^{2}(2 \pi f x)} .
\end{aligned}
$$




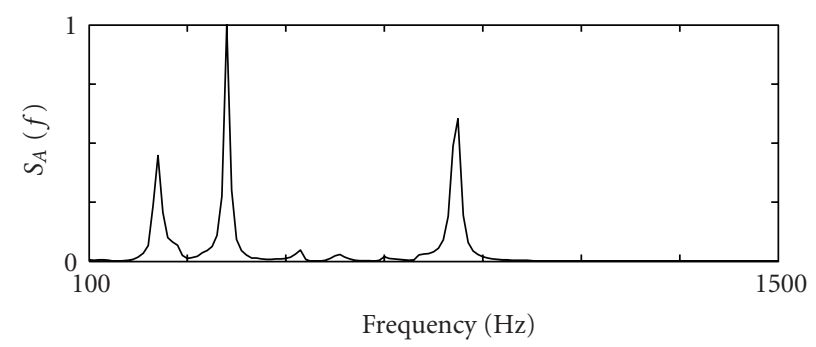

(a)

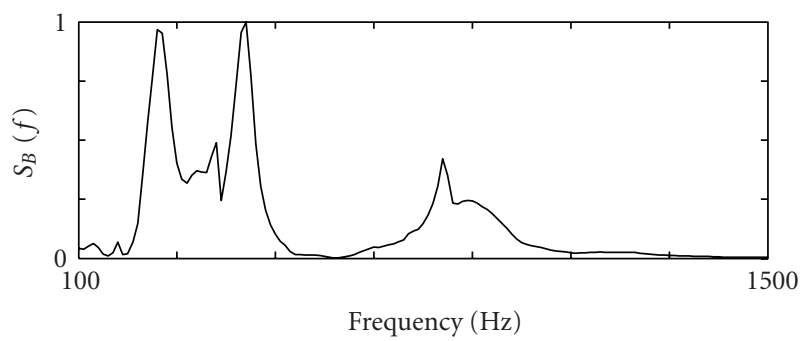

(b)

Figure 1: Power frequency responses of model structures A (a) and B (b).

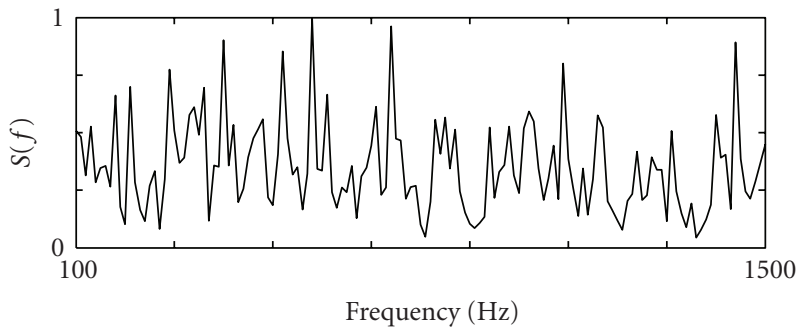

(a)

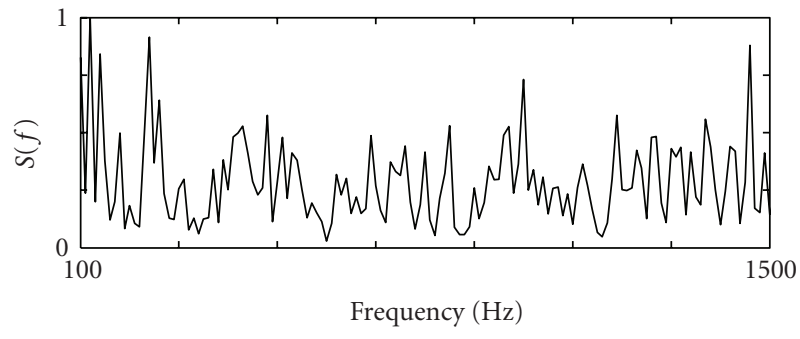

(b)

FIgURE 2: Examples of the clutter average spectrum of nonstationary vibration.

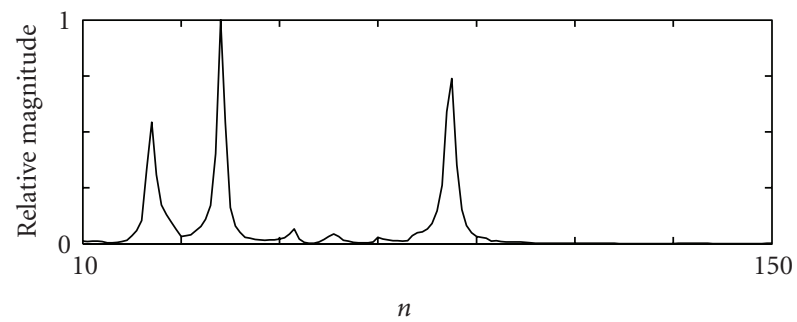

(a)

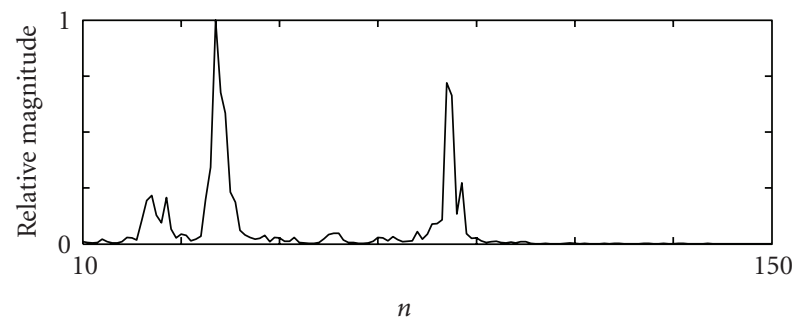

(c)

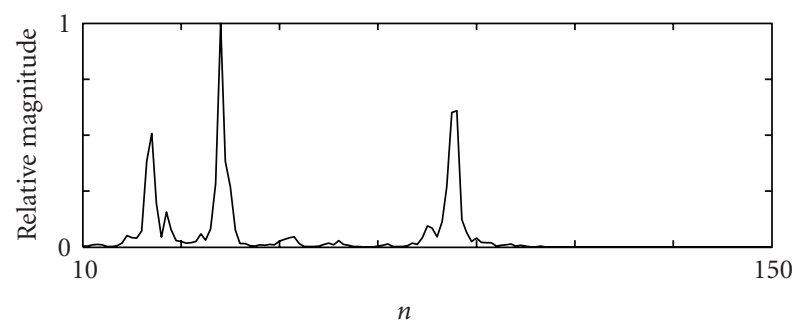

(e)

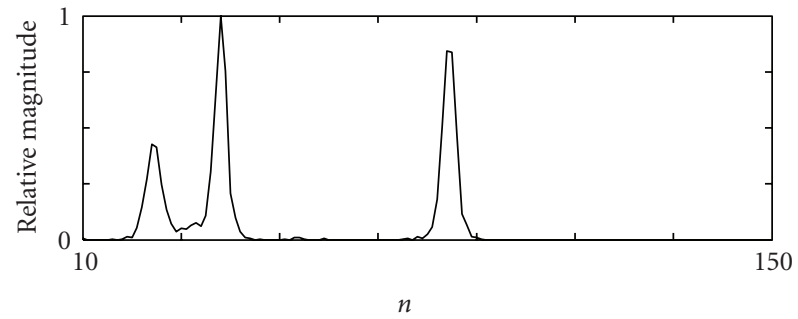

(b)

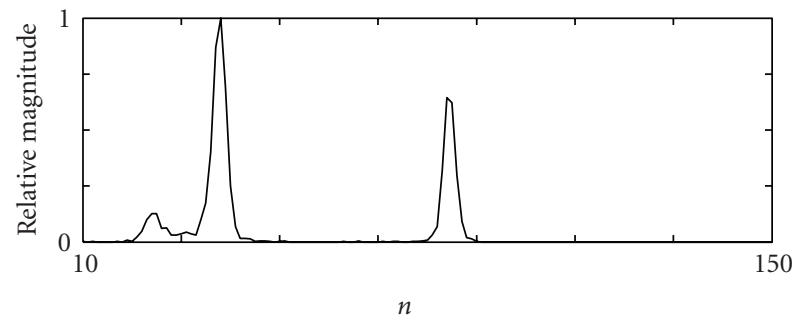

(d)

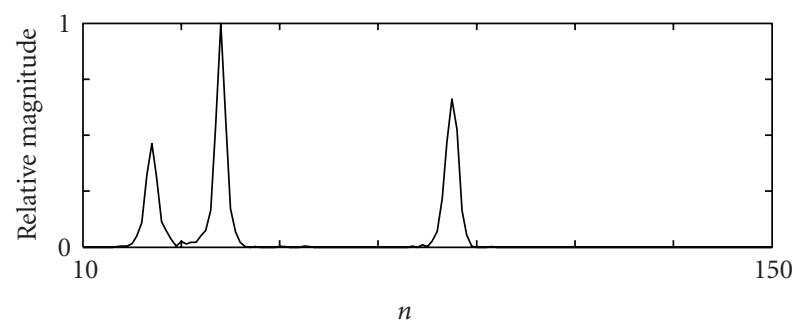

(f)

FIgure 3: Power frequency response of the model structure A estimated by the average DFT spectrum (a), (b), and (c) and the SIP distribution (b), (d), and (f). (a) and (b) Stationary random vibration. (c)-(f) Nonstationary vibration. Relative frequency is given by $n=f_{n} / 10$. 


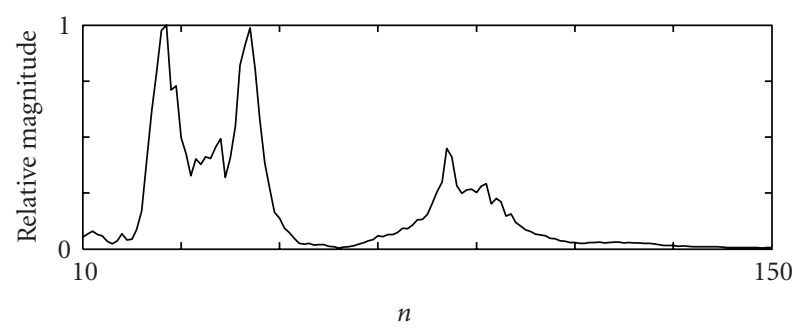

(a)

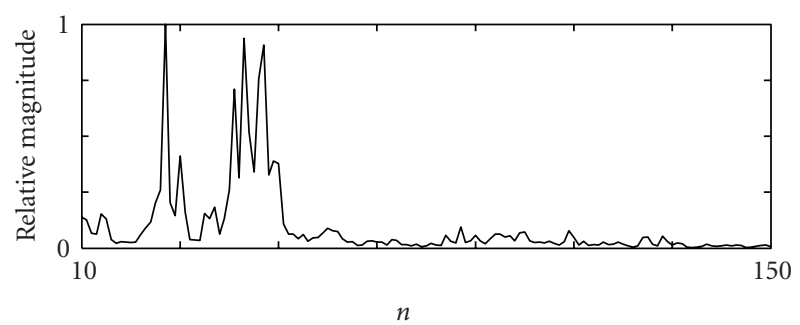

(c)

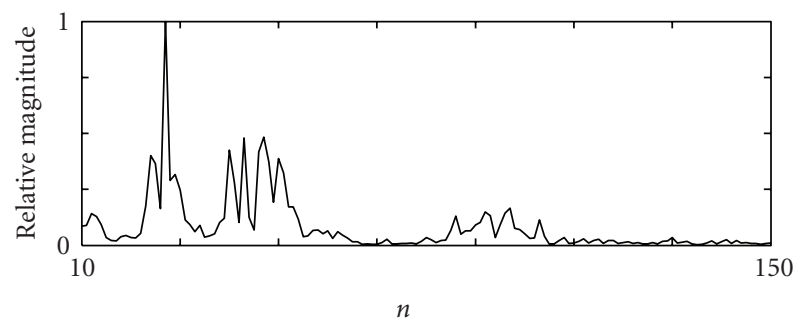

(e)

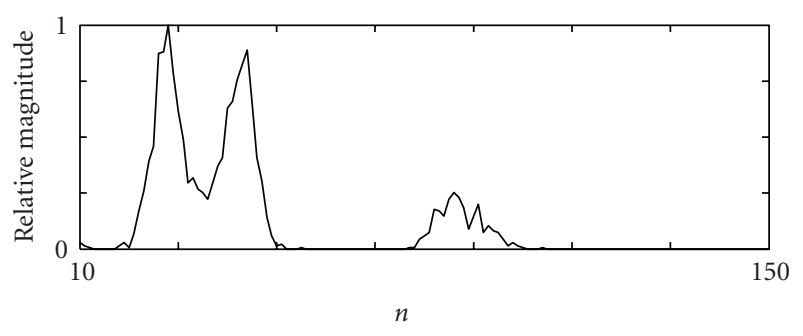

(b)

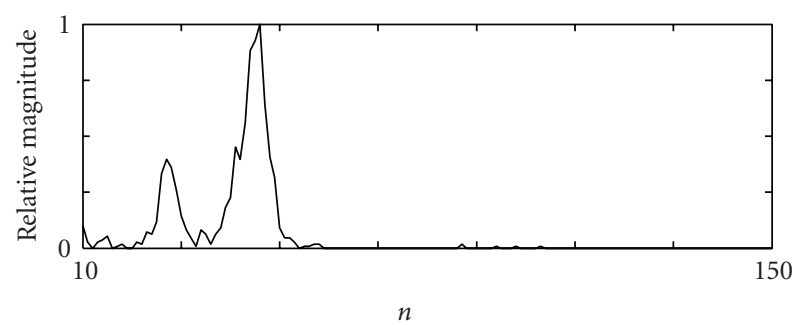

(d)

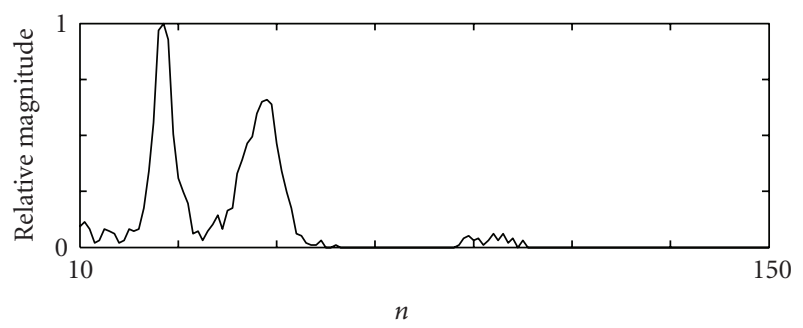

(f)

FIGURE 4: Power frequency response of the model structure B estimated by the average DFT spectrum (a), (c), and (e) and the SIP disctribution (b), (d), and (e). (a) and (b) Stationary random vibration. (c)-(f) Nonstationary vibration. Relative frequency is given by $n=f_{n} / 10$.

Hence, if we put

$$
\begin{gathered}
y(x, f)=a(f) \sin (2 \pi f x)+b(f) \cos (2 \pi f x), \\
Y(f)=\sum_{x=-M / 2}^{M / 2} y^{2}(x, f),
\end{gathered}
$$

we have the dominant frequency $f_{p}$ which satisfies

$$
Y\left(f_{p}\right)=\text { the maximum of } Y(f) \text {. }
$$

It should be noted that we attain a least squares fit of $W(x)$ to a sinusoid by $y\left(x, f_{p}\right)$.

The SIP distribution is given by a number of dominant frequencies (or periods) of short sequences which are fractions of measured data. Thus, the normalized SIP distribution $D\left(f_{p}\right)$ gives the probability of the dominant frequency $f_{p}$ found in measured data.

If we assume that a structure is excited by the force of random noise and assign the frequency $f$ in (1) such that

$$
f=f_{n}=n \Delta f, \quad n=1,2, \ldots, N
$$

where $\Delta f<1 / M$, we have, from [1],

$$
D\left(f_{j}\right)-D\left(f_{i}\right)=Q_{i j}(r) \frac{S\left(f_{j}\right)-S\left(f_{i}\right)}{S\left(f_{j}\right)+S\left(f_{i}\right)},
$$

where $0<Q_{i j}(r)<1$ and $S\left(f_{j}\right)$ is the power frequency response of a structure at a frequency $f_{j}$ and so on.

Hence, we get an approximation

$$
S\left(f_{n}\right) \approx k D\left(f_{n}\right)
$$

where $k$ is an appropriate constant. It should be mentioned that the spectral resolution given by $D\left(f_{n}\right)$ depends little on the length of the short sequence when $M f_{n}>1$.

\section{Experiment}

To confirm the theoretical result shown by (6) and apply the SIP distribution to the health monitoring of a structure, experiments were made using a model structure. A model structure is a wooden framework $(18(\mathrm{~W}) \times 22(\mathrm{D}) \times 27(\mathrm{H}) \mathrm{cm})$ which simulates a three-storied building with four struts. The strength of this model structure (the model structure A) is changed by altering the struts, that is, the cross-section 


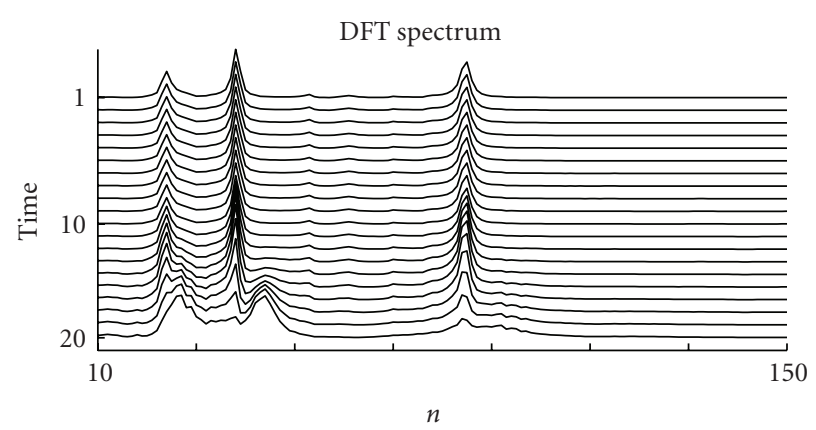

(a)

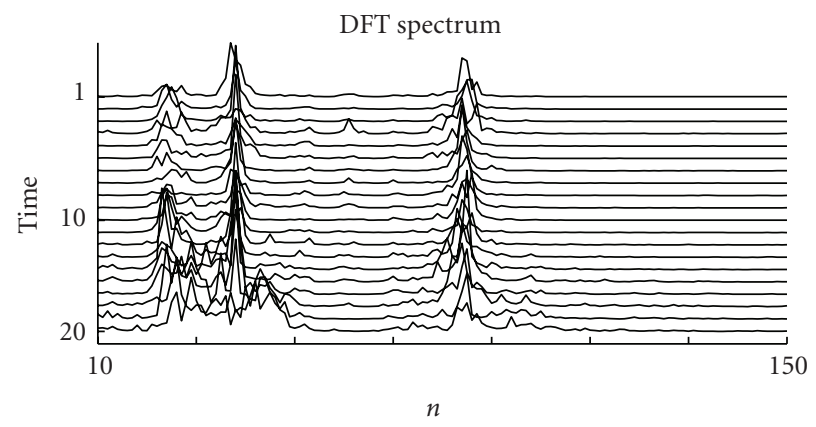

(c)

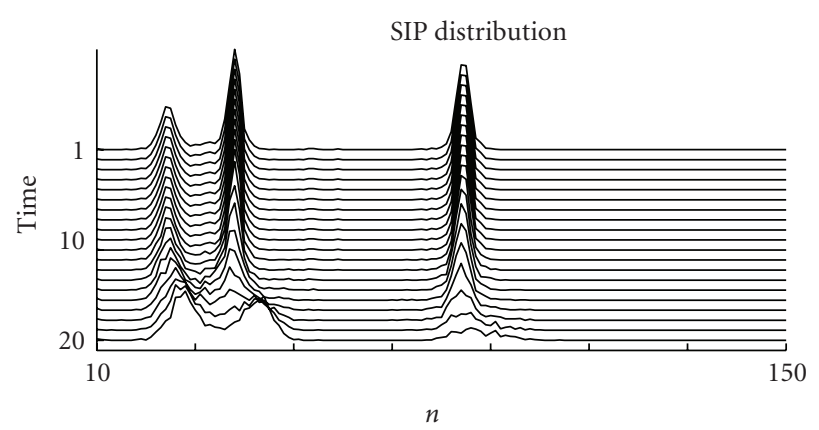

(b)

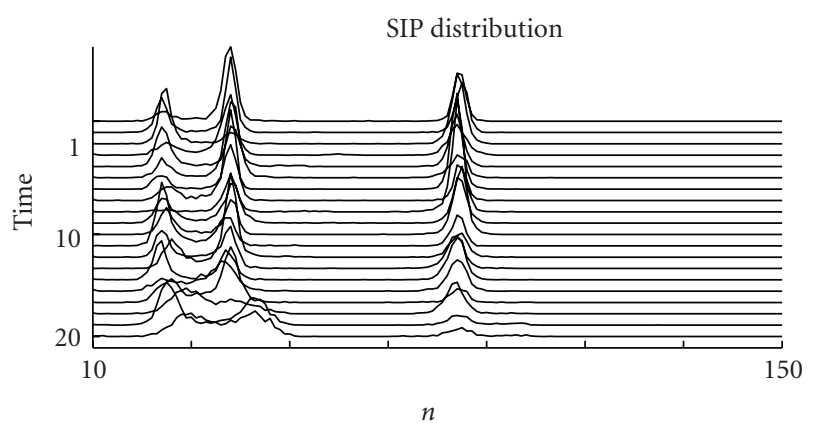

(d)

FIGURE 5: Illustration of the health-monitoring of a structure by the DFT spectrum and the SIP distribution for random noise excitation (a), (b) and nonstationary noise excitation (c), (d).

area of struts has a 25-percent decrease. This changed model structure (a model structure $\mathrm{B}$ ) is assumed to be the damaged structure of the model A.

The frequency responses of the model structures A and $B$, which are difficult to measure in real cases, are shown in Figure 1, where the power frequency response $S_{A}(f)$ and $S_{B}(f)$ are shown within the observation band.

The force of stationary random vibration as well as nonstationary one was applied to excite a model structure, and the acceleration of the structure was measured at the fixed point of the frame. The measured data were provided for the DFT analysis and SIP distribution.

We assume that the average spectrum of nonstationary vibration is a clutter one which varies slowly with time, so that we represent a nonstationary vibration as a set of random noise sequences each having a different clutter average spectrum; see Figure 2.

The measured data sequence was divided into 2,400 short-interval sequences for the nonharmonic Fourier analysis to get a SIP distribution, that is, the dominant frequency of each sequence is given by the analysis of 60 sampled data. To compare the SIP distribution with the average of DFT spectra, the measured data sequence was also divided into 240 sequences, so that the DFT frequency, $f_{n}$, is given by $n F / 600$, where $F$ is a sampling frequency. Corresponding to the DFT frequency, we put the frequency of the nonharmonic Fourier analysis (see (1)) such that $f=f_{n}$.

Figure 3 shows the power frequency response of the model structure A estimated by the average of 240 DFT spectra and the SIP distribution given by 2,400 dominant frequency samples. Both estimations are much the same when excited by the stationary random vibration, see Figures $3(a)$ and 3(b). Differences arise in the case of nonstationary vibrations, see Figures 3(c)-3(f).

Similarly, Figure 4 shows estimations of the power frequency response of the model structure B. We see that the SIP distribution is stable comparing with the DFT spectrum.

Figure 5 shows the illustration of the health-monitoring of a structure using its nonstationary vibration, where the model structure A changes gradually from time 10 and comes to the structure B at time 20. The DFT spectrum Figure 5(a) and the SIP distribution Figure 5(b) show the same change for random noise excitation. The difference of the both method arises when the structure is excited by nonstationary noises Figures 5(c) and 5(d). It seems that the SIP distribution is stable comparing with the DFT spectrum.

\section{Conclusions}

A number of buildings, bridges, and towers have been constructed in past decades. Consequently, there are many decrepit structures which need to be reconstructed. One may remember the accident in Minneapolis, USA where thirteen persons were killed by the sudden collapse of an old bridge over the Mississippi. The health monitoring of structures is an important mean for security against such an accident. The method described in this paper might be available. Further 
experiments using a real bridge, for example, will confirm the proposed method, though it will take decades.

\section{References}

[1] Y. Hirata, "Estimation of the frequency response of a structure using its non-stationary vibration," Journal of Sound and Vibration, vol. 313, no. 3-5, pp. 363-366, 2008.

[2] S. M. Kay and S. L. Marple Jr., "Spectrum analysis-a modern perspective," Proceedings of the IEEE, vol. 69, no. 11, pp. 13801419, 1981.

[3] Y. Hirata, "Non-harmonic Fourier analysis available for detecting very low-frequency components," Journal of Sound and Vibration, vol. 287, no. 3, pp. 611-613, 2005. 

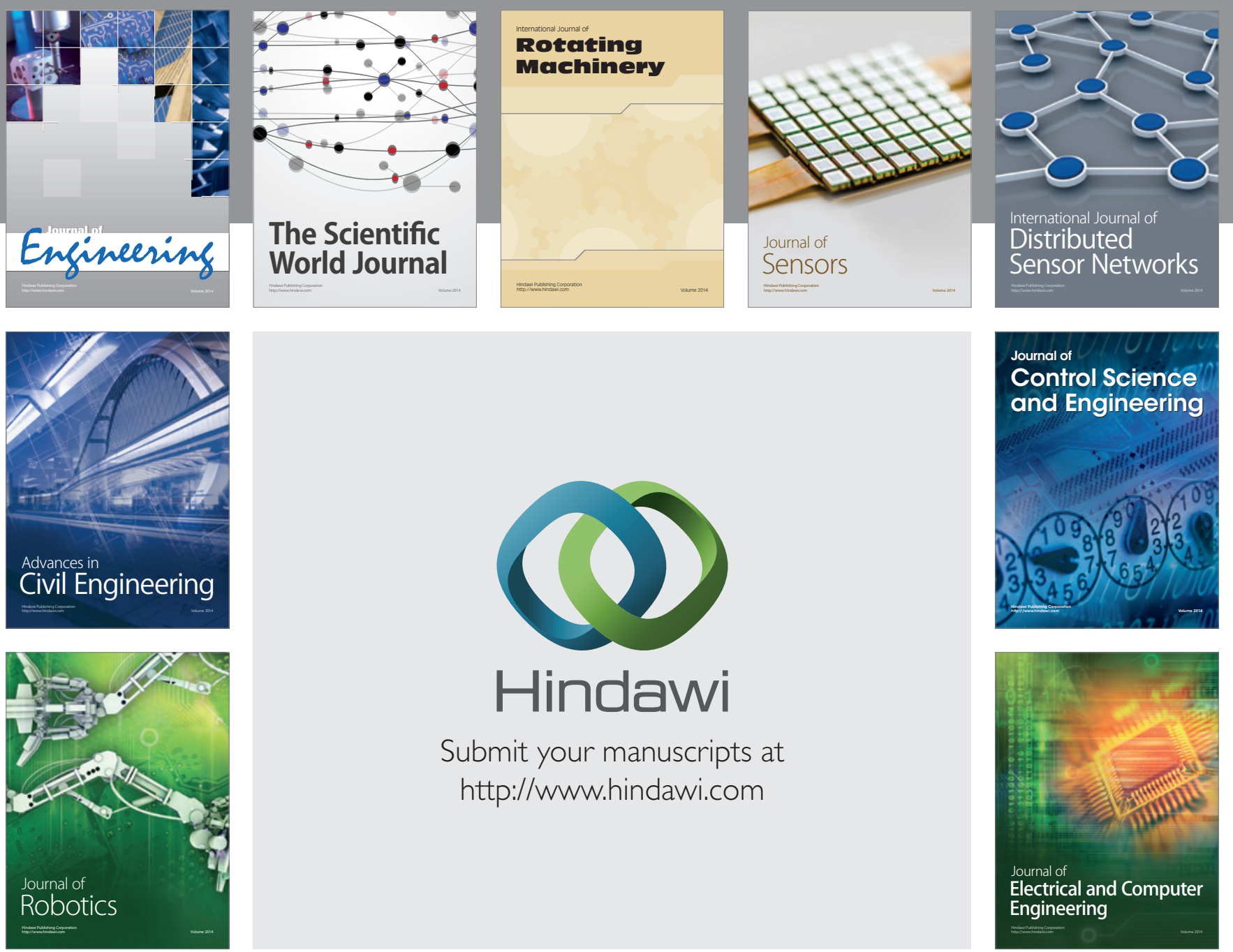

Submit your manuscripts at

http://www.hindawi.com
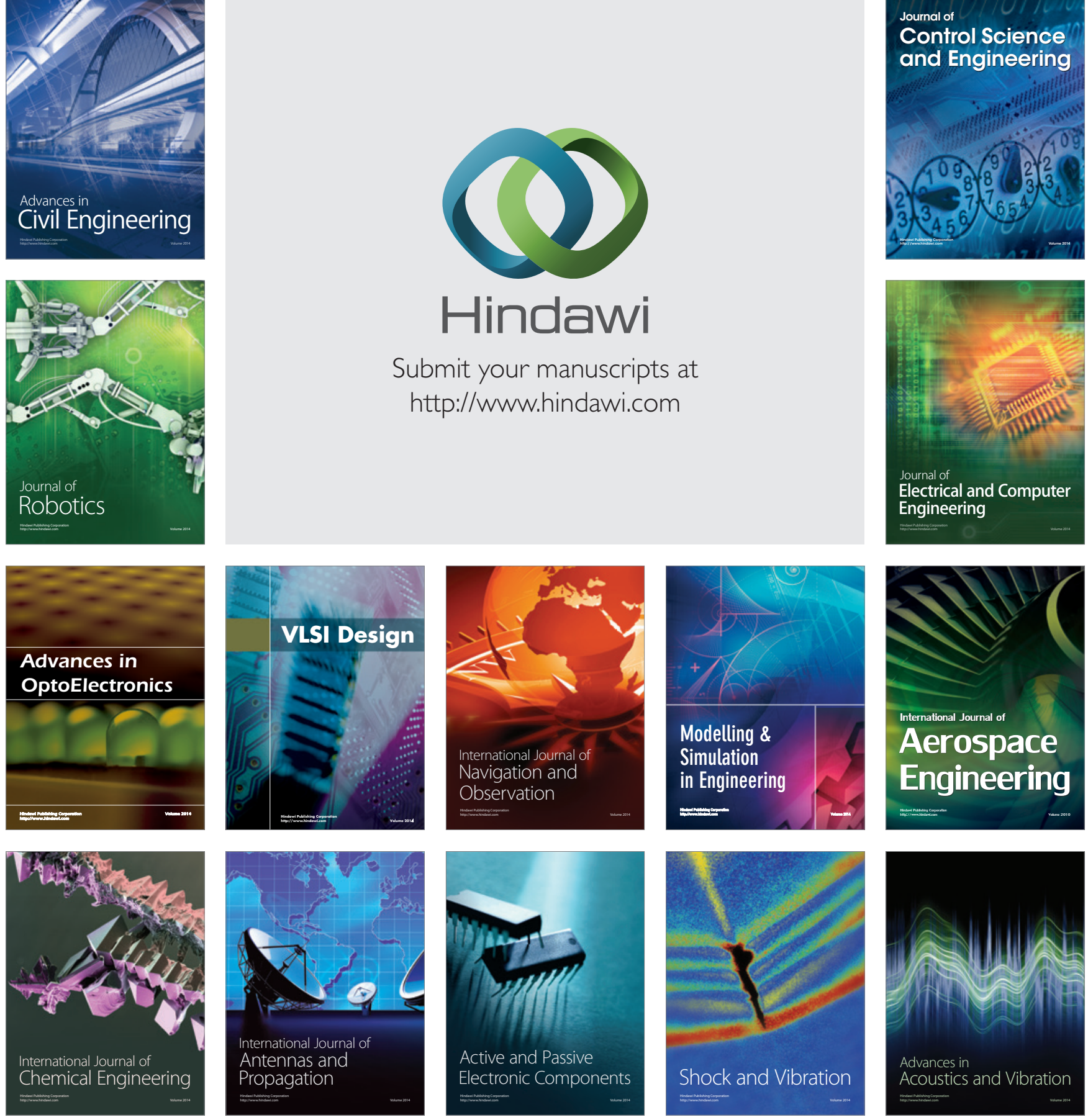